Andrew M. Biedermann ${ }^{1,2}$, Isabella R. Gengaro ${ }^{1,2}$, Sergio A. Rodriguez-Aponte ${ }^{2,3}$, Kerry R. Love $^{1,2}$, J. Christopher Love ${ }^{1,2}$

\title{
Modular development enables rapid design of media for alternative hosts
}

${ }^{1}$ Department of Chemical Engineering, Massachusetts Institute of Technology, Cambridge, Massachusetts 02139, United States

${ }^{2}$ The Koch Institute for Integrative Cancer Research, Massachusetts Institute of Technology, Cambridge, Massachusetts 01239, United States

${ }^{3}$ Department of Biological Engineering, Massachusetts Institute of Technology, Cambridge, Massachusetts 02139, United States

${ }^{*}$ Correspondence to: clove@mit.edu

Target journal: Biotechnology \& Bioengineering Article ( $<15$ double-spaced pages of text and no more than 10 figures/tables)

Keywords: Media development; media blending; Pichia pastoris; alternative hosts; automation 


\section{Abstract}

Developing media to sustain cell growth and production is an essential and ongoing

38 activity in bioprocess development. Modifications to media can often address host or product-

39 specific challenges, such as low productivity or poor product quality. For other applications,

40 systematic design of new media can facilitate the adoption of new industrially relevant

41 alternative hosts. Despite manifold existing methods, common approaches for optimization often

42 remain time and labor intensive. We present here a novel approach to conventional media

43 blending that leverages stable, simple, concentrated stock solutions to enable rapid

44 improvement of measurable phenotypes of interest. We applied this modular methodology to

45 generate high-performing media for two phenotypes of interest: biomass accumulation and

46 heterologous protein production, using high-throughput, milliliter-scale batch fermentations of

47 Pichia pastoris as a model system. In addition to these examples, we also created a flexible

48 open-source package for modular blending automation on a low-cost liquid handling system to

49 facilitate wide use of this method. Our modular blending method enables rapid, flexible media

50 development, requiring minimal labor investment and prior knowledge of the host organism, and

51 should enable developing improved media for other hosts and phenotypes of interest. 


\section{Introduction}

Achieving high volumetric productivities of biologic drugs in cultivation is a key step in

54 advancing candidate biologic drugs. The outcome of this effort ultimately impacts

55 manufacturing costs as well as readiness for transitioning clinical-stage development (Love,

56 Love, \& Barone, 2012). The development of standard, chemically defined media for established

57 manufacturing hosts, such as $\mathrm{CHO}$, has made such transitions efficient for monoclonal

58 antibodies by achieving high biomass accumulation, cell viability, operational consistency, and

59 specific productivities, streamlining development efforts (McGillicuddy, Floris, Albrecht, \&

60 Bones, 2018; Rodrigues, Costa, Henriques, Azeredo, \& Oliveira, 2012). Nonetheless, optimizing

61 productivity or quality attributes for a specific product often still requires further refinement of

62 media (Ritacco, Wu, \& Khetan, 2018). Such development may require evaluating dozens of

63 variants derived from a common standard formulation to address the specific challenges

64 encountered (Gagnon et al., 2011; Loebrich et al., 2019). Media development for entirely new

65 biomanufacturing technologies, such as alternative hosts (Matthews, Kuo, Love, \& Love, 2017a)

or new product modalities (Lu et al., 2016), may also require new formulations or extensive

67 optimizations due to limited prior knowledge.

Common approaches to develop a medium to optimize a phenotype of interest are often

69 labor intensive, low throughput, or rely heavily on extensive analytical capacity (Galbraith,

70 Bhatia, Liu, \& Yoon, 2018). For example, analysis of residual media after cultivation requires

71 extensive capabilities for analytical characterization and prior experience with the manufacturing

72 host to identify potentially limiting or toxic media components (Mohmad-Saberi et al., 2013;

73 Pereira, Kildegaard, \& Andersen, 2018). As a result, optimizations can be slow and iterative.

74 Furthermore, for an alternative host such as Komagataella phaffii (formerly known as Pichia

75 pastoris), there is substantially less, if any, prior knowledge available to establish profiles for

76 residual components in media after fermentation. Other analytical techniques like RNA-seq

77 combined with methods for reporter metabolite analysis can guide media optimization, to 
generate testable hypotheses regarding beneficial modifications to media (Matthews et al., 2017a). Such genome-scale approaches, however, require prior host-specific knowledge, such as well-annotated genomes, and are still limited by slow iteration and labor-intensive

81 preparations of new media to test the hypotheses generated from computational analyses.

Alternative strategies for blending basal components for media allow linear combinations

83 of existing media to explore many variations rapidly (Jordan, Voisard, Berthoud, \& Tercier,

84 2013). While this approach avoids slow iterative analyses, the typical experiment is labor

85 intensive to perform, often requiring independent preparations of over a dozen stock media to

86 combine (Rouiller et al., 2013). Similar to analytical-based approaches for optimization, the

87 selected variations of media are simultaneously guided and constrained by prior experience and

88 media designs, which may limit the breadth of components examined (Kennedy \& Krouse,

89 1999). For less established hosts with fewer available formulations of media, media blending

90 may also require fully de novo formulations for initial studies. Further complicating such designs,

91 different and new components for media can present challenges in solubility or unanticipated

92 interactions with other elements in the formulations (Ritacco et al., 2018). New approaches to

93 blending could, however, enable fast, flexible experimentation and minimize the time, labor, and

94 analytical development needed initially to optimize media for new applications and phenotypes.

95 Here, we present a novel and generalizable approach for the modular development of

96 media and demonstrate its use to create optimized media for two different phenotypes—cellular

97 growth and recombinant expression of a protein (as measured by the secreted heterologous

98 protein titer) from Pichia pastoris. Our approach comprises two modular parts for blending and

99 optimization. We determined that a set of simple concentrated stock solutions constructed in

100 defined modules could generate many media by blending or dilution. We then automated a

101 simple, inexpensive liquid handling system (Opentrons OT-2) to enable high-throughput

102 screening for the effects of diverse media on a phenotype of interest in milliliter-scale batch

103 cultures. To maximize the benefit of this automated blending, we also developed an algorithmic 
104 framework for systematic modular media optimization, beginning from a simple minimal media

105 (here a YNB-based one). This framework provides insights pertaining to key media components

106 during stages of optimization, as well as overall mapping of the design space for the media. In

107 the examples presented here, the resulting defined media developed with this strategy

108 outperformed commonly used BMGY and BMMY complex formulations for biomass

109 accumulation and secreted heterologous protein production.

\section{Materials and Methods}

\section{Strains and cultivation conditions}

Media for evaluating biomass accumulation were developed using a previously

described strain expressing G-CSF under control of the pAOX1 promoter (Crowell et al., 2020).

24-well plate screens were conducted as described previously, except cells were grown on a

labForce shaker and were only sampled 24 hours after inoculation (Matthews et al., 2017a).

117 BMGY, BMMY, and RDM media were formulated as described previously for shake flasks

118 (Matthews et al., 2017a). All cultivations were inoculated from a working cell bank at an initial

119 cell density of $0.1 \mathrm{OD} / \mathrm{mL}$. For each working cell bank, cells grown in $1 \mathrm{~L}$ shake flasks with a 200

$120 \mathrm{~mL}$ working volume of RDM were harvested during exponential growth (4-5.5 OD/mL) via

121 centrifugation at $1500 \mathrm{rcf}$ for 4 minutes at $23^{\circ} \mathrm{C}$ and resuspended in an equal volume mixture of

$122 \mathrm{RDM}$ and $50 \mathrm{v} / \mathrm{v} \%$ glycerol. This mixture was then distributed into $700 \mu \mathrm{L}$ aliquots and stored at

$123-80{ }^{\circ} \mathrm{C}$, resulting in a cell density of $\sim 30 \mathrm{OD} / \mathrm{mL}$ for the cell bank.

$124 \quad$ Media for evaluating enhanced production were developed using a strain expressing a

125 rotavirus-derived subunit vaccine candidate, $\mathrm{P}[8]$, under the control of the pAOX1 promoter

126 described previously (Dalvie et al., 2020). Biomass accumulation proceeded for 24 hours; cells

127 reached an initial induction density of $\sim 20 \mathrm{OD} / \mathrm{mL}$. Cultures were then exchanged into

128 production media and allowed to produce protein for an additional 24 hours. Supernatant was

129 harvested by centrifugation at $1500 \mathrm{rcf}$ for 4 minutes at $23^{\circ} \mathrm{C}$ and filtered using a Captiva 96 
well $0.2 \mu \mathrm{m}$ filter plate (Agilent Technologies, Santa Clara, CA) prior to titer measurement by RP-UHPLC.

Media components and supplements were purchased from Sigma-Aldrich, St. Louis,

$133 \mathrm{MO}$, unless otherwise indicated in the supporting information. A table of supplement and stock

134 solutions with screening concentrations is also included in the supporting information. During

135 modular optimization, all media were prepared in high throughput using an Opentrons OT-2

136 liquid handler (Opentrons, Brooklyn, NY, software version $\geq 3.16 .1$ ) using Openblend. Modular

137 media blending code and instructions for setup and operation are provided in the Openblend package (https://github.com/abiedermann/openblend_public). For consistency, media used in final head-to-head comparisons were prepared in bulk and filter sterilized through a $0.2 \mu \mathrm{m}$ benchtop filter.

\section{Analytical procedures}

Biomass was measured by optical density at $600 \mathrm{~nm}$ as described previously (Matthews et al., 2017a). An Agilent Bravo liquid handler was used to dilute samples prior to 145 measurements of OD into the Tecan Infinite M200 Pro plate reader.

147 performed on Agilent 1290 Infinity II UHPLC system controlled using OpenLab CDS software

148 (Agilent Technologies, Santa Clara, CA). The concentration of protein was determined using a

149 Poroshell $120 \mathrm{SB}-\mathrm{Aq}$ column $(2.1 \times 50 \mathrm{~mm}, 1.9 \mu \mathrm{m})$ operated at $1.0 \mathrm{~mL} / \mathrm{min}$ and $70{ }^{\circ} \mathrm{C}$ (Agilent

150 Technologies, Santa Clara, CA). Buffer A was $0.1 \%(\mathrm{v} / \mathrm{v})$ TFA in water and buffer B was $0.1 \%$

151 (v/v) TFA, 0.5\% (v/v) water in ACN. A gradient was performed as follows: 30\% B for 1 min., 30-

$15240 \%$ B over 3 min., 40-90\% B over 0.5 min., $90 \%$ B for 0.5 min., $90-30 \% B$ over 0.5 min., and

$15330 \%$ for $1 \mathrm{~min}$; ; total method run time was 6.5 minutes. Sample injection volumes were $50 \mu \mathrm{L}$. A

154 diode array detector was set for absorbance detection at $214 \mathrm{~nm}$. Data analysis was completed 155 using OpenLab CDS Data Analysis (Agilent Technologies, Santa Clara, CA). 

$>0.01$ ) were eliminated sequentially in order of decreasing adjusted $p$-value to avoid overfitting. Data was plotted using Prism 8.4.0 (GraphPad Software, San Diego, CA).

\section{Results}

164 Design of approach for modular media blending

We sought to develop an approach capable of identifying important, beneficial

modifications for media tailored to a given phenotype of interest. We reasoned that key

167 requirements for such an approach would be that it is fast and automatable, with minimal dependence on complex analytical assay development. Such features would enable routine application to any measurable phenotype of interest. In general, media blending allows both speed and low analytical complexity. We aimed to retain these features while minimizing the

171 labor and constraints on compositions imposed by linear combinations of fully formed and

172 unique media. We reasoned that diverse and flexible blends of media could be created by

173 defining simple concentrated stock solutions as basic modules to combine further. These

174 modules would comprise individual components or common subsets of components with

175 compatible solubilities (e.g. YNB). If media components could be formulated in concentrated

176 stock solutions that could be stored stably over time, then the components could be routinely

177 and interchangeably combined and diluted to the desired final concentrations. This approach

178 would yield a broadly applicable modular strategy for media blending amenable to conventional 179 liquid handling automation.

To test the feasibility of this approach, we first assessed whether many common media 
$\mathrm{CHO}$ medium eRDF as a reference, we estimated the solubility of each component of this medium, using data from AqSoIDB as well as other online sources (Combs, 2012; FSA Panel on Additives and Products or Substances used in Animal Feed (FEEDAP), 2011; Ritacco et al., 2018; Schnellbaecher, Binder, Bellmaine, \& Zimmer, 2019; Sorkun, Khetan, \& Er, 2019;

Yamamoto \& Ishihara, n.d.). We compared the estimated solubility of each media component to

187 its concentration in eRDF and found that, individually, most media components are soluble at

188 levels $>10 x$ higher than their eRDF concentration (Figure 1A). The existence of a wide range of

189 commercially available concentrated supplements further supports this result: $>50 x$

190 concentrated solutions of amino acids, vitamins, lipids, and trace metal supplements are

191 common and commercially available.

Next, we used the product information of commercially available supplements, literature sources, and inspection to estimate the percentage of eRDF media components that could be stored in stable solutions for $>6$ months. We estimated that over $75 \%$ of eRDF components met this criterion (Figure 1B). To address stability challenges caused by less stable components, we reasoned that less stable components or supplements, such as vitamins, could be prepared, 197 aliquoted, and stored frozen for long-term storage (Schnellbaecher et al., 2019); these aliquots could then be thawed and used within a defined period to mitigate component instability and enable their integration into our modular blending strategy. Together, these solubility and stability data suggested that a modular approach to media development could be defined in this way to accommodate a range of new formulations easily. chose this liquid handler due to its low cost, reliability, and compatibility with simple formats for

204 data input, such as Excel spreadsheets. We then created an open-source Python package,

205 named Openblend, which simplified the media construction process by handling routine

206 experimental design and execution steps (Figure 1C). Openblend creates an experimental

207 design spreadsheet, specifying the number of 24 well plates, the desired media composition of 
each well, and stock solution names and concentrations. The script then checks the feasibility of the experimental design, ensuring that the total volume of each well will not exceed the target volume and avoiding the addition of sub-microliter stock solution volumes. If the design passes

211 this assessment, the script then outputs a new spreadsheet containing the setup for the OT-2

212 deck and required volumes of stock solutions, providing a user with instructions on how to setup

213 the OT-2 liquid handler. We found that our typical time to execute this script, setup the OT-2

214 and initiate plate building was $\sim 15$ minutes, and the time for the automated steps was about two 215 hours.

216 Finally, we defined a modular approach for optimization to effectively leverage the

217 Openblend tool (Figure 1D). Beginning from an initial basal medium, improved media are 218 constructed through successive rounds of optimization. In each round, a library of media 219 components and supplements are screened to identify beneficial additives. These additives are

220 then screened in combination and over a range of concentrations to further optimize the

221 performance of the medium. Each modular addition and optimization of additives can be guided

222 simply by measurements of the phenotype of interest (e.g. biomass accumulation). This greedy

223 approach to multi-dimensional optimization could continue iteratively until the resulting media

224 met desired specifications, all available media components were explored, or no additional 225 gains in performance realized.

To assess the utility of this blending-based approach, we next aimed to identify and 229 optimize the concentration of media components beneficial for rapid biomass accumulation of $P$. 230 pastoris in batch cultivation. We previously described a rich defined medium (RDM) (Matthews 231 et al., 2017a), capable of high growth rates during biomass accumulation. One challenge 232 encountered with this formulation, however, was that precipitates can form at higher $\mathrm{pH}$ values 233 that require filtering during bulk preparations. Nonetheless, this medium provided a relevant 
234 comparison for assessing the medium realized with our new approach due to its prior

235 demonstrated benefits relative to complex media. Following our modular approach, we

236 improved biomass accumulation by optimizing the accumulated optical density at $600 \mathrm{~nm}$ after

23724 hours of cultivation.

Algorithms for optimizing systems based on multiple dimensions are often sensitive to 239 initial conditions used (Zakharova \& Minashina, 2015). Given this potential confounding effect

240 here, we tested first the effects of the types of carbon source, nitrogen source, and pH set point

241 on biomass accumulation, using 1x YNB without amino acids or ammonium sulfate (YNB) to

242 satisfy minimum requirements for the concentrations of trace elements. We conducted a full-

243 factorial DOE using glycerol, glucose, and fructose as carbon sources; urea and ammonium

244 sulfate as nitrogen sources; and potassium phosphate as a buffer with pH values of $5,5.75$, and

245 6.5. We selected initial concentrations of $40 \mathrm{~g} / \mathrm{L}, 4 \mathrm{~g} / \mathrm{L}$ urea or the $\mathrm{N}-\mathrm{mol}$ equivalent for

246 ammonium sulfate, and $10 \mathrm{~g} / \mathrm{L}$ potassium phosphate, similar to values used in other media for

247 Pichia pastoris (Matthews et al., 2017a). A least squares regression model, including individual,

248 combination, and quadratic effects was fit to the log of optical density after 24 hours, a proxy

249 variable for the average growth rate $\left(R^{2}=0.81\right)$. We determined that the two most significant

250 model terms were the type of carbon source and the interaction of the nitrogen source with $\mathrm{pH}$

251 (Figure 2A). We found that cells grew significantly faster on metabolically related sugars

252 (glucose and fructose) than on the polyol (glycerol) commonly used for Pichia during biomass

253 accumulation (Figure 2B). This result affirms prior reports where glucose has been used for

254 biomass accumulation of Pichia (Guo et al., 2012; Moser et al., 2017).

255

The model also suggested that poor biomass accumulation during cultivation resulted

256 from a combination of ammonium sulfate as a source of nitrogen with low buffer pH (Figure

257 2B). This outcome may result from the production of acidic species associated with cellular

258 ammonium metabolism in the batch cultivation (Villadsen, 2015). Interestingly, the model

259 indicated slightly greater biomass was achieved with urea instead of ammonium sulfate. The 
biomass accumulation of cultures grown with urea as a source of nitrogen were less sensitive to

261 reduced $\mathrm{pH}$ values $(\sim 5)$. We observed, however, that cultivations at $\mathrm{pH} 5$ showed extensive

262 flocculation compared to those at 6.5. Given the insensitivity of urea-fed cultivations to buffer

$263 \mathrm{pH}$ and the high solubility and potential for low-cost sourcing of fructose, we therefore chose to

264 include fructose, urea, and a potassium phosphate buffer with a $\mathrm{pH}$ of 6.5 in our initial media

265 formulation.

With this basal formulation determined, we next screened for concentration-dependent

267 interactions of other key additives to the media and then optimized concentration-dependent

268 parameters. Following the same approach for screening effects, we conducted a full factorial

269 DOE over a broad range of media component concentrations: YNB $(0.5,1,2 x)$, fructose $(10,30$,

$27050 \mathrm{~g} / \mathrm{L})$, urea $(1,4,7 \mathrm{~g} / \mathrm{L})$, and potassium phosphate adjusted to a $\mathrm{pH}$ of $6.5(4,10,16 \mathrm{~g} / \mathrm{L})$. The

271 resulting model identified fructose as a concentration-sensitive parameter $\left(R^{2}=0.73\right)$ (Figure

272 2D). Terms involving the concentration of YNB were also highly ranked, but not statistically

273 significant. No significant interactions between components were identified in the model. We

274 therefore sought to better understand the concentration dependence of fructose and YNB

275 independently (Figure 2E), over an 8-fold range of concentrations. As expected, biomass

276 accumulation was highly sensitive to fructose concentration, with an optimum around $22.5 \mathrm{~g} / \mathrm{L}$ of

277 fructose. The concentration of YNB had minimal effect on biomass accumulation; the presence

278 of trace elements supplied by YNB, however, was essential to growth. Based on these results,

279 we chose concentrations of $22.5 \mathrm{~g} / \mathrm{L}$ fructose, $1 \times \mathrm{YNB}, 7 \mathrm{~g} / \mathrm{L}$ urea, and $10 \mathrm{~g} / \mathrm{L}$ potassium

280 phosphate buffer. We reasoned that although biomass accumulation was relatively insensitive

281 to the concentrations of YNB and urea, higher concentrations could provide improved media

282 depth in future applications. We named this basal formulation DM1_dev0.

283 We next assessed what additional media components could improve biomass

284 accumulation. To test over 60 different components individually would require over 60 individual

285 solutions. Such an approach would scale linearly with new components; instead, we chose to 
screen groups of related components, using commercially available pre-mixed supplements. We compiled a library of 16 commercial supplements and industrially-relevant surfactants containing more than 60 unique components and screened their individual effect on biomass accumulation after 24 hours. In this way, we reasoned we could efficiently identify critical classes of components related to the phenotype of interest and potentially deconvolve specific individual

291 additives of interest by inference. We used the recommended concentrations of each

292 supplement as supplied in product information, or critical micelle concentrations, and prior

293 knowledge for broad classes in yeast media to set reasonable screening concentrations

294 (Supporting Information). We identified five beneficial and two detrimental supplements that 295 significantly impacted biomass accumulation ( $p_{a d j}<0.02 ; 1$-way-ANOVA) (Figure 2F). In 296 general, the results suggest that supplementation with amino acids and trace metals were 297 beneficial for accumulating biomass, while two surfactants, Tween 20 and CHAPS, were 298 detrimental. For this phenotype, the effects of vitamin and lipid supplements were minor; supplements from either supplement category were not significantly beneficial or detrimental to biomass accumulation. Our earlier experiments suggest that vitamins are essential but concentration agnostic (Figure 1E), while lipid supplementation provides no clear benefit for

302 biomass accumulation.

Based on these results, we chose to test whether combinations of supplements of amino

304 acids and trace salts could yield synergistic improvements in biomass accumulation. We 305 screened pairwise combinations of the five beneficial supplements of mixed composition and 306 ranked the performance of our supplementation strategies (Figure 2G). A combination of 1x 307 MEM amino acids with $0.1 \mathrm{v} / \mathrm{v} \%$ PTM1 salts resulted in the highest yield of biomass, though we 308 observed strong performance from other combinations of amino acid and trace metal 309 supplements. Based on these data, we chose to add MEM amino acids and PTM1 salts in our 310 basal medium and optimized their concentrations (Figure 2H). 
Based on these results, we elected $0.1 \mathrm{v} / \mathrm{v} \%$ PTM1 salts and $1 \mathrm{x}$ MEM amino acids, in

312 order to balance the moderate benefits and potentially high costs of amino acids. We found,

313 however, that the inclusion of the PTM1 salts in liter-scale preparations produced fine

314 precipitates, which can impede sterile transfers in use. To overcome this challenge, we

315 screened a broad range of PTM1 salts concentrations to identify the minimum concentration

316 required for improved outgrowth performance (Figure 2I). We found that PTM1 addition at

317 concentrations as low as $0.0005 \mathrm{v} / \mathrm{v} \%$ led to increased biomass accumulation. We therefore

318 revised our PTM1 salts concentration to $0.01 \mathrm{v} / \mathrm{v} \%$, a concentration high enough to obtain the

319 benefits of PTM1 supplementation without inducing precipitate formation. This formulation we

320 named DM1.

321 Completing this series of optimizations with our iterative modular approach to define a

322 new formulation of medium, we then compared with other common media used to grow $P$.

323 pastoris. We evaluated the performance of this new optimized medium (DM1) relative to the

324 unsupplemented basal medium (DM1_dev0), the rich defined medium (RDM) we had previously

325 developed, and a common medium 4 v/v\% glycerol BMGY. We found that DM1 yielded the

326 highest biomass accumulation, with significantly higher biomass accumulation relative to RDM

327 and BMGY (Figure 2J). This result demonstrates the utility of our modular strategy here for

328 media development that yielded an improved formulation for biomass accumulation compared

329 to other common media with minimal time and labor investment, and without requiring complex

330 analytical methods like mass spectrometry or RNA-sequencing.

Identifying media conditions important to heterologous protein production in $\mathrm{K}$. phaffii

In addition to the time and labor savings of modular media development, our proof-of-

334 concept experiments demonstrated that this approach creates a flexible medium that can be

335 rapidly adapted to new growth phenotypes, as well as a data package that the identifies media

336 conditions important to the phenotype of interest. We reasoned that these additional benefits 
could be particularly relevant for optimizing production of heterologous proteins. Understanding which media components contribute most significantly to productivity could improve culture performance and help identify important metabolic pathways or physiological effects for further study.

To develop a medium for improved production of a recombinant protein, we chose to use

342 a strain engineered to secrete a rotavirus-derived subunit vaccine component, VP4-P[8], as a

343 model protein. We have previously demonstrated that this viral antigen can be expressed at

344 high titer under the control of the methanol-inducible pAOX1 promoter in BMMY media (Dalvie

345 et al., 2020). Similar to our initial approach to optimize a medium for growing biomass, we first

346 determined and optimized the concentrations of the sources for carbon and nitrogen, along with

347 the $\mathrm{pH}$. The expression of $\mathrm{P}[8]$ in the strain tested uses the methanol-dependent $\mathrm{pAOX} 1$

348 promoter for inducible expression, so we selected methanol as the initial carbon source. We

349 then examined the impact of the source of nitrogen and buffer $\mathrm{pH}$ on titer. We conducted a full-

350 factorial DOE using identical concentrations as those used to create a medium for accumulating

351 biomass. The resulting model was visualized by ranking combinations of sources of nitrogen

352 and buffer (Figure 3A). The effects showed no interaction between these two factors. Urea was

353 again identified as the preferred source of nitrogen while higher $\mathrm{pH}$ values led to improved

354 secreted $\mathrm{P}[8]$ productivity. Unlike biomass accumulation, this $\mathrm{pH}$ dependence was observed

355 across both nitrogen sources.

We next applied the same DOE to identify important concentration-dependent

357 interactions that impact the production of P[8]. Unsurprisingly, the concentration of methanol

358 was the most important factor, with possible minor effects from other components (Figure 3B).

359 We decided to screen further a 20 -fold range in methanol concentrations using two formulations

360 for remaining media components - the one determined for optimal cell growth (DM1) and the

361 optimal base media formulation predicted by the quadratic model here (2x YNB, $1 \mathrm{~g} / \mathrm{L}$ urea, 4

$362 \mathrm{~g} / \mathrm{L}$ potassium phosphate adjusted to a $\mathrm{pH}$ of 6.5$)$. We found that production was relatively 
insensitive for concentrations of methanol ranging from $1-4 \mathrm{v} / \mathrm{v} \%$, with an optimum around $2 \%$

(Figure $3 \mathbf{C}$ ). We postulated that the rapid decline in productivity observed in these milliliterscale cultures using concentrations $>6 \mathrm{v} / \mathrm{v} \%$ methanol was likely due to excess formation of toxic metabolic byproducts such as formaldehyde and hydrogen peroxide (Wakayama et al., 2016). Interestingly, the predicted optimal medium from this set of studies outperformed the medium we determined for accumulating biomass, suggesting that certain components of the basal medium may benefit protein expression more than cellular growth and underscores the value of optimizing media for specific phenotypes of interest. Based on these data in total, we

371 defined a basal medium for production including 2x YNB, $2 \mathrm{v} / \mathrm{v} \%$ methanol, $1 \mathrm{~g} / \mathrm{L}$ urea, and 4 372 g/L potassium phosphate buffer adjusted to a pH of 6.5 (DM2_dev0).

We added three chemical chaperones (TUDCA, sodium deoxycholate monohydrate (SDM), and valproic acid) (Kuryatov, Mukherjee, \& Lindstrom, 2013; Uppala, Gani, \& Ramaiah, 2017), two antioxidants (reduced glutathione (GSH) and N-acetyl cysteine (NAC)), and the chelator, K-

377 ETDA, to the list of 16 supplements included in our original screen defined for biomass accumulation. Concentrations for these components were chosen based on product specifications, literature data, and prior experience (Supporting Information). Many of the 22 supplements screened improved production of P[8] (Figure 3D). The top four ranking

381 supplements comprised surfactants or lipids, which could modulate membrane fluidity and lipid 382 metabolism (Butler, Huzel, Barnab, Gray, \& Bajno, 1999; Degreif, Cucu, Budin, Thiel, \& Bertl, 383 2019; Ritacco, Frank V; Yongqi Wu, 2018).

We then screened combinations of lipid supplements and surfactants to identify potential synergistic effects. We ranked the individual supplements and their combinations (Figure 3E) according to the measured titers of $P[8]$. We found that the addition of a cholesterol-rich

387 supplement yielded the highest secreted titers of $P[8]$ ( $50 \%$ improvement compared with 
alone did not substantially improve performance, suggesting the benefit results from a combination of fatty acids and surfactant components in the supplement (Supporting Information). This conclusion is consistent with similar improvements observed from other supplements, such as linoleic acid-oleic acid-albumin (Figure 3D). assessed the dependence of titer on the concentration of the cholesterol-containing supplement identified (Figure 3F). Similar to our observations with cellular YNB used in the outgrowth 396 media, we found that concentrations of the supplement as low as $0.2 \mathrm{v} / \mathrm{v} \%$ were beneficial for 397 protein expression, but that production was relatively insensitive to concentration (Figures 3F, 398 3G). We then directly compared the supplemented medium to the original composition; the new supplemented media provided a $25 \%$ improvement in titer $(p=0.0006$, one-tailed Welch's T test). This new formulation with $1 \mathrm{x}$ cholesterol supplement, which we named DM2_dev1, was the result of one cycle of optimization using our method.

407 (Figure 3H). We, therefore, considered if there could be additional classes of beneficial 408 supplements, absent from the original screen. Previous experiments demonstrated that $P[8]$ 409 productivity is highly sensitive to methanol concentration (Figure 3C), so we wondered whether 410 further modulation of central carbon metabolism could yield additional productivity gains. Modification of central carbon metabolism is best accomplished by feeding cells

412 alternative carbon sources, either entirely or as co-feeding substrates. Four co-fed substrates

413 have previously been shown to be non-repressive of pAOX1: sorbitol, mannitol, trehalose, and 414 alanine (Inan \& Meagher, 2001). These substrates can be co-utilized with methanol without 
415 repressing the pAOX1 promoter, which controls expression of P[8]. We hypothesized that the

416 introduction of supplemental carbon sources could enable further optimization of central carbon

417 metabolism. We screened co-fed substrates individually and in 1:1 combinations at a total

418 concentration of $20 \mathrm{~g} / \mathrm{L}$ (a concentration similar to the optimal fructose and methanol

419 concentrations observed in previous carbon source optimizations) (Figure 2E,3C). Sorbitol co-

420 feeding had the most beneficial effect, resulting in a 80\% increase in P[8] titer (Figure 3I).

421 Mannitol supplementation was also beneficial ( $70 \%$ increase), while alanine and trehalose co-

422 feeding were detrimental to productivity. While co-feeding carbon sources led to increased

423 biomass yield during production, these differences did not account for the improved titer, as

424 improvements in specific productivity $\left(q_{p}\right)$ of $\sim 60 \%$ and $\sim 45 \%$ were also observed for the sorbitol

425 and mannitol co-fed conditions, respectively (Supporting Information). Based on these data,

426 we chose to include sorbitol as a supplemental carbon source for further study.

427 The addition of a supplemental carbon source could significantly impact central carbon

428 metabolism. We, therefore, wondered how the inclusion of sorbitol might impact the optimal

429 carbon feeding strategy. Examining total carbon source concentrations from $20-70 \mathrm{~g} / \mathrm{L}$, we

430 compared the performance of cultures co-fed with sorbitol:methanol ratios of $3: 1,1: 1$, and $1: 3$ to

431 a methanol-only control (Figure 3J). All co-fed conditions outperformed the methanol-only

432 control, suggesting that the presence of sorbitol is highly beneficial for producing P[8]. The titer

433 was relatively insensitive to sorbitol:methanol ratios and carbon concentrations. Based on the

434 data, we decided to use $2 \mathrm{v} / \mathrm{v} \%$ methanol and $20 \mathrm{~g} / \mathrm{L}$ of sorbitol for the final sorbitol-

435 supplemented media named DM2.

436 Finally, we compared the P[8] titer obtained using DM2_dev0, DM2_dev1, and DM2 to

437 other common production media for $P$. pastoris: BMMY and RDM. We found that DM2 led to a

$438 \sim 2 x$ improvement in P[8] titers, relative to BMMY and RDM, up to $97 \pm 2 \mathrm{mg} / \mathrm{L}$. 
Here we have implemented a novel and broadly applicable approach for media

442

443

444

445

446

447

448

449

450

451

452

453

454

Our optimized formulations affirmed the importance of lipid-related components for

maximizing titers in Pichia pastoris cultivations. The importance of optimizing membrane fluidity

or lipid metabolism has been well established in $\mathrm{CHO}$ and appears to be key to optimizing

heterologous protein secretion in P. pastoris cultivation as well (Clincke et al., n.d.; Ritacco et

al., 2018; Zhang, Wang, \& Liu, 2013).

Modular media blending has four advantages over existing methods. First, the use of common stock solutions and supplements to formulate media reduces initial labor required for new experiments or optimizations $\sim 15$ minutes per experiment, making parallel testing of multiple hypotheses efficient and requires less resources overall. Here, we created 30 stock solutions, and evaluated $>360$ unique media compositions, without manual preparation of individual media or extensive blending calculations or planning. Most of these solutions could be readily reused in future experiments to optimize for new phenotypes of interest. Second, our method requires minimal knowledge of the host organism a priori and could, in principle, be applied to any measurable phenotype of interest. We anticipate that this method could be used to optimize other phenotypes of interest, such as glycosylation profiles. Third, our method 
challenges. These traits make it possible to learn about formulations that may lead to extensive precipitates like those encountered with our rich defined medium formulation (Figure 4A). Finally, modularly constructed media, such as DM2, can be $\sim 70 \%$ pure water with low osmolarity, leaving volumetric and osmotic space for future modifications to accommodate new

471 or related phenotypes of interest (Figure 4B).

We also acknowledge certain limitations in the present study that may be addressed in

473 future work. First, while modular media development identifies components key to the

474 optimization of the phenotype of interest, additional media optimization effort may be necessary

475 to translate these learning in batch cultivations to scaled-up fed-batch or perfusion operation,

476 where additional variables such as supplemental feed composition and feeding schedule must

477 also be considered. In principle, modular media construction could be applied to high-

478 throughput scale-down cultivation models, such as Ambr250s. Second, our approach for

479 optimization relies on greedy algorithms tailored to create a new media for a single phenotype of

480 interest; however, given the vast explorable media space it is possible to find a local optimum.

481 Further metabolic or -omic modeling techniques could be employed to guide broader exploration

482 of media space, co-optimize multiple phenotypes, or facilitate biologically informed optimization,

483 albeit with more complex experimental and computational requirements (Matthews, Kuo, Love,

484 \& Love, 2017b; Mohmad-Saberi et al., 2013). Third, our current method used commercially

485 available supplements, but in practice, beneficial supplements could be simplified by using

486 individual components, to facilitate more biological inferences and aid development of improved

487 host-specific supplements. Finally, initial screens to identify beneficial supplements rely on

488 reasonable choices of initial concentrations for screening. These currently require prior

489 knowledge from the literature or commercial sources; with further use in the community of the

490 Openblend approach, it is possible additional sharing of knowledge could help inform further

491 developments. 
The improved speed and accessibility of in-depth media development experiments enabled by modular media construction could help improve expression of many classes of

494 proteins in laboratories and discovery centers that have not traditionally had access to such

495 capabilities. Since many lead candidates for new therapeutic proteins begin in small biotech

496 firms and academic labs, early-stage improvements in productivity could help advance more

497 proteins towards the clinic simply by facilitating access to larger quantities of proteins for initial

498 research and non-clinical studies. In more established companies, the ability to make rapid

499 improvements to existing media may enable faster product development timelines and could

500 reduce manufacturing costs overall. Rapid identification and optimization of sensitive media

501 components could also enable easier adoption of a range of industrially relevant alternative

502 hosts, resulting in further manufacturing flexibility and potentially cost savings (Coleman, 2020).

Acknowledgements

The authors acknowledge Danielle Camp for program coordination. This work was funded by the Bill \& Melinda Gates Foundation (Investment ID INV-002740). The content is solely the responsibility of the authors and does not necessarily represent the official views of the Bill \& Melinda Gates Foundation.

A.M.B., I.R.G., K.R.L., and J.C.L. conceived and planned experiments. A.M.B. conducted media development experiments. S.R.A. developed and maintained the RP-UPLC

511 assay. A.M.B. performed analytical characterization. A.M.B., K.R.L., and J.C.L. wrote the

512 manuscript. J.C.L. and K.R.L. designed the experimental strategy and supervised analysis. All 513 authors reviewed the manuscript. 


\section{References}

Butler, M., Huzel, N., Barnab, N., Gray, T., \& Bajno, L. (1999). Linoleic acid improves the robustness of cells in agitated cultures, 27-36.

Clincke, M.-F., Guedon, E., Yen, F. T., Ea, L., Universite, N., Ogier, V., ... Goergen, J.-L. (n.d.). Effect of Surfactant Pluronic F-68 on CHO Cell Growth, Metabolism , Production, and Glycosylation of Human Recombinant IFN- c in Mild Operating Conditions, 181-190. https://doi.org/10.1002/btpr.503

Coleman, E. (2020). Establishment of a Novel Pichia Pastoris Host Production Platform by.

Combs, G. F. (2012). The vitamins: Fundamental aspects in nutrition and health. San Diego: Elsevier Academic Press.

Cooper, R. A. (1978). Influence of Increased Membrane Cholesterol on Membrane Fluidity and Cell Function in Human Red Blood Cells. Journal of Supramolecular Structure.

Crowell, L. E., Crowell, L. E., Raymond, A., St, H. E., Engineering, C., Doyle, P. S., \& Crowell, L. E. (2020). Accelerating process development for biologics on an automated, pharmacyscale manufacturing system by by.

Dalvie, N. C., Brady, J. R., Crowell, L. E., Tracey, M. K., Biedermann, A. M., Kaur, K., ... Love, J. C. (2020). Molecular engineering improves antigen quality and enables integrated manufacturing of a trivalent subunit vaccine candidate for rotavirus. BioRx, 1-51.

Degreif, D., Cucu, B., Budin, I., Thiel, G., \& Bertl, A. (2019). Lipid determinants of endocytosis and exocytosis in budding yeast. BBA - Molecular and Cell Biology of Lipids, 1864(7), 1005-1016. https://doi.org/10.1016/j.bbalip.2019.03.007

FSA Panel on Additives and Products or Substances used in Animal Feed (FEEDAP). (2011). Scientific Opinion on safety and efficacy of choline chloride as a feed additive for all animal species. EFSA Journal, 9(9), 2353.

Gagnon, M., Hiller, G., Luan, Y. T., Kittredge, A., Defelice, J., \& Drapeau, D. (2011). High-End $\mathrm{pH}$-controlled delivery of glucose effectively suppresses lactate accumulation in $\mathrm{CHO}$ Fedbatch cultures. Biotechnology and Bioengineering, 108(6), 1328-1337. https://doi.org/10.1002/bit.23072

Galbraith, S. C., Bhatia, H., Liu, H., \& Yoon, S. (2018). Media formulation optimization: current and future opportunities. Current Opinion in Chemical Engineering, 22, 42-47. https://doi.org/10.1016/j.coche.2018.08.004

Guo, C., Huang, Y., Zheng, H., Tang, L., He, J., Xiang, L., ... Jiang, H. (2012). Secretion and activity of antimicrobial peptide cecropin D expressed in Pichia pastoris. Experimental and Therapeutic Medicine, 1063-1068. https://doi.org/10.3892/etm.2012.719

Inan, M., \& Meagher, M. M. (2001). Non-repressing carbon sources for alcohol oxidase (AOX1) Promoter of Pichia pastoris, 92(6), 585-589.

Jordan, M., Voisard, D., Berthoud, A., \& Tercier, L. (2013). Cell culture medium improvement by rigorous shuffling of components using media blending, 31-40. https://doi.org/10.1007/s10616-012-9462-1

Kennedy, M., \& Krouse, D. (1999). Strategies for improving fermentation medium performance: a review. Journal of Industrial Microbiology and Biotechnology, (23), 456-475.

Kuryatov, A., Mukherjee, J., \& Lindstrom, J. (2013). Chemical Chaperones Exceed the Chaperone Effects of RIC-3 in Promoting Assembly of Functional a 7 AChRs. PLoS ONE, 8(4), 1-11. https://doi.org/10.1371/journal.pone.0062246

Loebrich, S., Clark, E., Ladd, K., Takahashi, S., Brousseau, A., Kitchener, S., ... Ryll, T. (2019). Comprehensive manipulation of glycosylation profiles across development scales. MAbs, 11(2), 335-349. https://doi.org/10.1080/19420862.2018.1527665

Love, J. C., Love, K. R., \& Barone, P. W. (2012). Enabling global access to high-quality biopharmaceuticals. Current Opinion in Chemical Engineering, 2(4), 383-390. https://doi.org/10.1016/j.coche.2013.09.002 
598

599

600

601

602

603

604

605

606

607

608

609

610

611

612

613

614

615

616

617
Lu, T. L., Pugach, O., Somerville, R., Rosenberg, S. A., Kochenderfer, J. N., Better, M., \& Feldman, S. A. (2016). A Rapid Cell Expansion Process for Production of Engineered Autologous CAR-T Cell Therapies. Human Gene Therapy Methods, 27(6), 209-219. https://doi.org/10.1089/hgtb.2016.120

Mahammad, S., \& Parmryd, I. (2015). Cholesterol depletion using methyl- $\beta$-cyclodextrin. In Methods in membrane lipids (pp. 91-102).

Matthews, C. B., Kuo, A., Love, K. R., \& Love, J. C. (2017a). Development of a general defined medium for Pichia pastoris, (July), 103-113. https://doi.org/10.1002/bit.26440

Matthews, C. B., Kuo, A., Love, K. R., \& Love, J. C. (2017b). Development of a general defined medium for Pichia pastoris, (July), 103-113. https://doi.org/10.1002/bit.26440

McGillicuddy, N., Floris, P., Albrecht, S., \& Bones, J. (2018). Examining the sources of variability in cell culture media used for biopharmaceutical production. Biotechnology Letters, 40(1), 5-21. https://doi.org/10.1007/s10529-017-2437-8

Mohmad-Saberi, S. E., Hashim, Y. Z. H. Y., Mel, M., Amid, A., Ahmad-Raus, R., \& PackeerMohamed, V. (2013). Metabolomics profiling of extracellular metabolites in CHO-K1 cells cultured in different types of growth media. Cytotechnology, 65(4), 577-586. https://doi.org/10.1007/s10616-012-9508-4

Moser, J. W., Prielhofer, R., Gerner, S. M., Graf, A. B., Wilson, I. B. H., Mattanovich, D., \& Dragosits, M. (2017). Implications of evolutionary engineering for growth and recombinant protein production in methanol - based growth media in the yeast Pichia pastoris. Microbial Cell Factories, 1-16. https://doi.org/10.1186/s12934-017-0661-5

Pereira, S., Kildegaard, H. F., \& Andersen, M. R. (2018). Impact of CHO Metabolism on Cell Growth and Protein Production: An Overview of Toxic and Inhibiting Metabolites and Nutrients. Biotechnology Journal, 13(3), 1-13. https://doi.org/10.1002/biot.201700499

Ritacco, Frank V; Yongqi Wu, A. K. (2018). Cell Culture Media for Recombinant Protein Expression in Chinese Hamster Ovary ( $\mathrm{CHO}$ ) Cells : History, Key Components, and Optimization Strategies. https://doi.org/10.1002/btpr.2706

Ritacco, F. V., Wu, Y., \& Khetan, A. (2018). Cell culture media for recombinant protein expression in Chinese hamster ovary $(\mathrm{CHO})$ cells: History, key components, and optimization strategies. Biotechnology Progress, 34(6), 1407-1426. https://doi.org/10.1002/btpr.2706

Rodrigues, M. E., Costa, A. R., Henriques, M., Azeredo, J., \& Oliveira, R. (2012). Comparison of commercial serum-free media for $\mathrm{CHO}-\mathrm{K} 1$ cell growth and monoclonal antibody production. International Journal of Pharmaceutics, 437(1-2), 303-305. https://doi.org/10.1016/j.ijpharm.2012.08.002

Rouiller, Y., Périlleux, A., Collet, N., Jordan, M., Stettler, M., \& Broly, H. (2013). A highthroughput media design approach for high performance mammalian fed-batch cultures, (June), 501-511.

Schnellbaecher, A., Binder, D., Bellmaine, S., \& Zimmer, A. (2019). Vitamins in cell culture media: Stability and stabilization strategies. Biotechnology and Bioengineering, 116(6), 1537-1555. https://doi.org/10.1002/bit.26942

Sorkun, M. C., Khetan, A., \& Er, S. (2019). AqSolDB , a curated reference set of aqueous solubility and 2D descriptors for a diverse set of compounds, 2019(July), 1-8. https://doi.org/10.1038/s41597-019-0151-1

Uppala, J. K., Gani, A. R., \& Ramaiah, K. V. A. (2017). Chemical chaperone, TUDCA unlike PBA, mitigates protein aggregation efficiently and resists ER and non-ER stress induced HepG2 cell death. Scientific, 1(January), 1-13. https://doi.org/10.1038/s41598-017-039401

Villadsen, J. (2015). Redox Balances and Consistency Check of Experiments. In Fundamental Bioengineering (pp. 17-38).

Wakayama, K., Yamaguchi, S., Takeuchi, A., Mizumura, T., Ozawa, S., Tomizuka, N., ... 

metabolism of the methylotrophic yeast Pichia methanolica. Journal of Bioscience and Bioengineering, 122(5), 545-549. https://doi.org/10.1016/j.jbiosc.2016.03.022

Yamamoto, T., \& Ishihara, K. (n.d.). Stability of Glutathione in Solution. Developments in Food Engineering, 209-211.

Zakharova, E. M., \& Minashina, I. K. (2015). Review of Multidimensional Optimization Methods. Mathematical Models, Computational Methods, 60(6), 625-636. https://doi.org/10.1134/S1064226915060194

Zhang, H., Wang, H., \& Liu, M. (2013). Rational development of a serum-free medium and fedbatch process for a GS-CHO cell line expressing recombinant antibody, 363-378.

630 https://doi.org/10.1007/s10616-012-9488-4 


\section{A}

\section{Solubility / medium concentration for $\mathrm{CHO}$ media components}

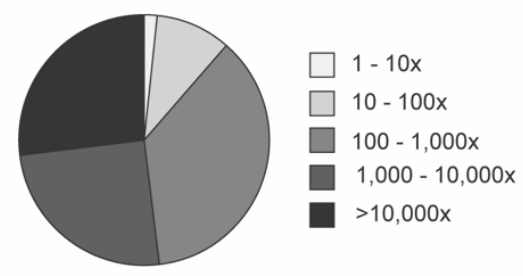

B

\section{Solution stability of} CHO media components

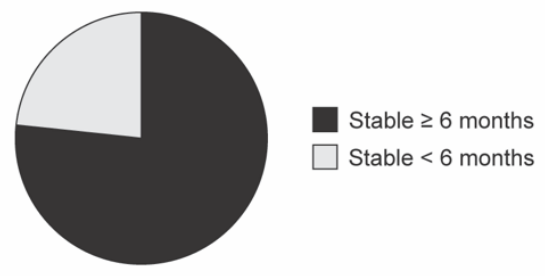

\section{Modular media optimization}

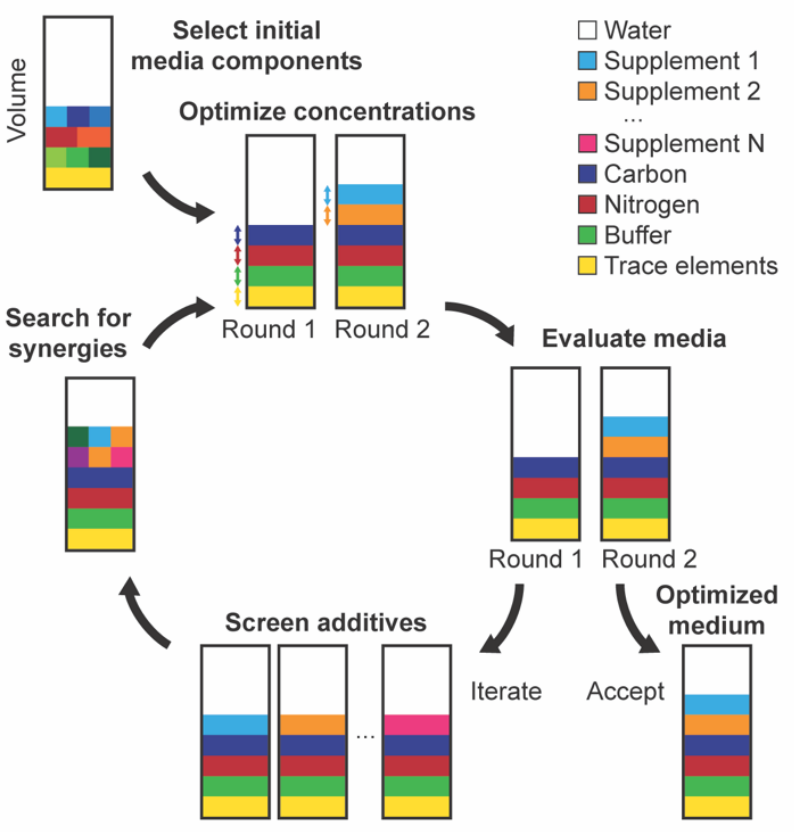

C Modular media blending

Add any new reusable stocks to library

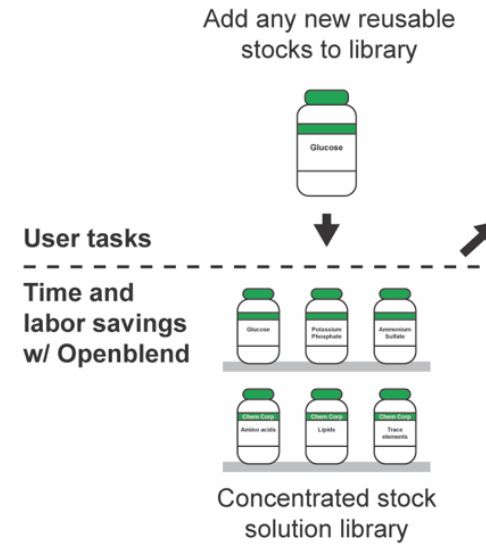

Input experimental design in spreadsheet

Pour stocks from library and follow setup deck

Test impact of diverse media conditions on phenotype of interest

Figure 1. Modular media development can be broadly applicable, easily applied, and systematically executed to improve measurable phenotypes of interest.

A) Estimate of the ratios of component solubility to their concentrations in medium demonstrates that most components are soluble at $>10 x$ their concentration in the $\mathrm{CHO}$ medium, eRDF. B) With the exception of some classes of medium components, such as vitamins, most media components can be formulated into solutions that remain stable for $>6$ months under proper storage conditions. C) Overview of time, labor and planning saved by using Openblend to automate modular media construction. D) Overview of a modular media optimization approach, 
641 systematically.

642

A

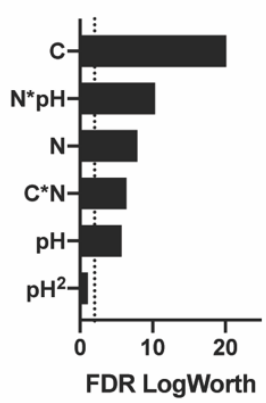

E
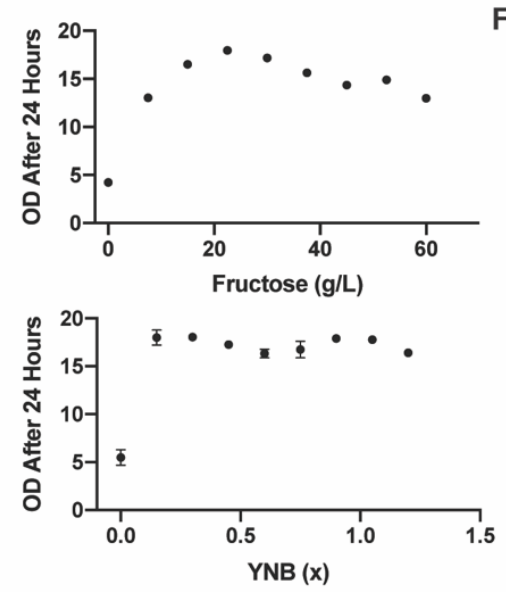

H

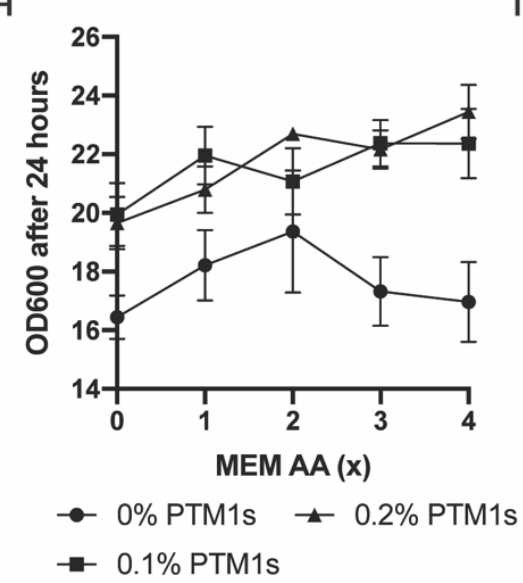

B

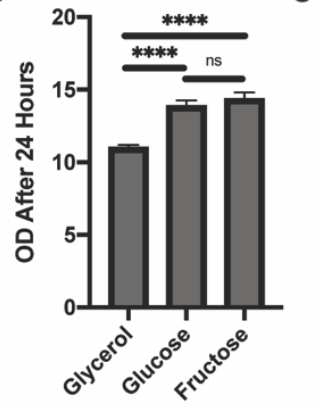

$\mathbf{F}$
C

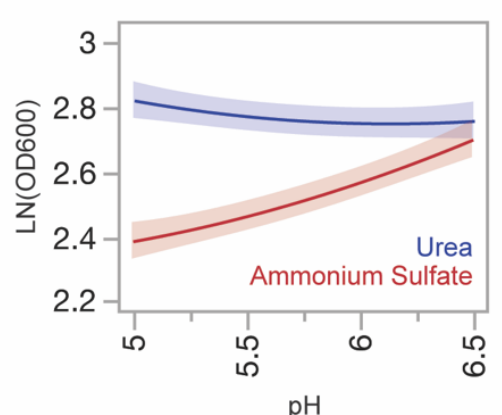

D

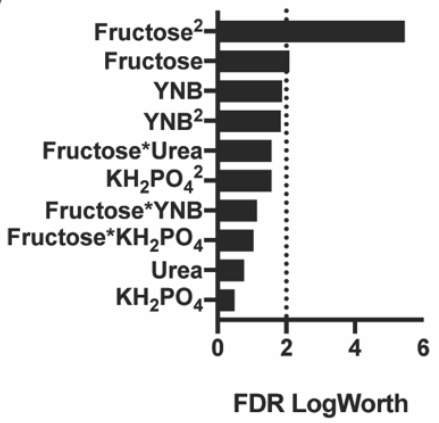

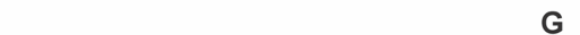

G

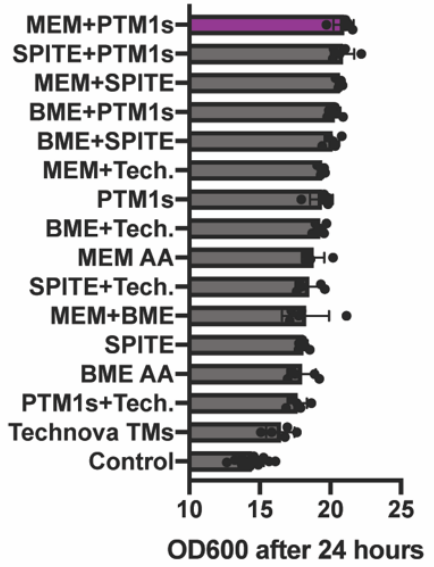

OD600 after 24 hours
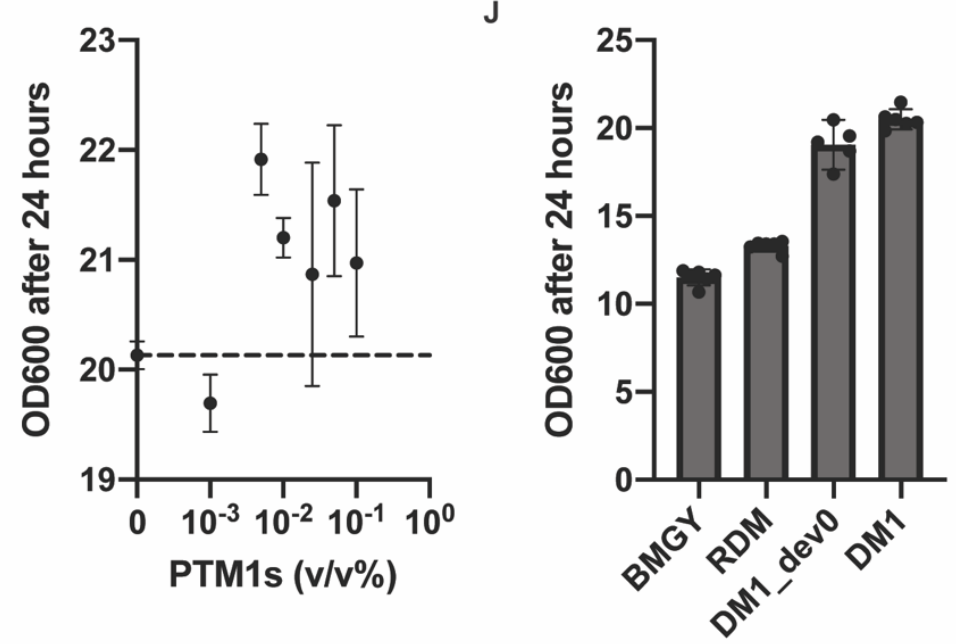

643

644

645

646

647

Figure 2. Modular development of a new biomass accumulation media for $P$. pastoris

A) Significance of carbon (fructose, glucose, glycerol), nitrogen (urea and ammonium sulfate), and $\mathrm{pH}$ choice $(5,5.75,6.5)$ in a least square regression model fitted to a full factorial DOE. B) Fructose and glucose were found to result in significantly higher biomass accumulation after 24 
648 hours of outgrowth than glycerol. C) Ammonium sulfate was found to be more $\mathrm{pH}$ sensitive than

649 urea, as shown by the JMP sensitivity profiles during fructose feeding. D) Significance of terms

650 in a least square regression model fitted to a full factorial DOE over fructose, urea, potassium

651 phosphate, and YNB concentrations. E) 1-FAAT optimization of fructose and YNB concentration

652 finds optimal outgrowth performance at a fructose concentration of $22.5 \mathrm{~g} / \mathrm{L}$ and relative

653 insensitivity over a wide range of YNB concentrations (0.15 to $1.2 x)$. F) A media

654 supplementation screen identified 5 beneficial supplements, related to trace element and amino

655 acid supplementation. G) Further screening of beneficial supplement combinations identified

656 synergistic amino acid and trace metal supplementation strategies. H) Comparison of the effect

657 of MEM amino acid concentration on biomass accumulation at different PTM1 salts

658 concentrations. I) Effect of the concentration of PTM1 salts on biomass accumulation in

659 DM1_dev0 medium supplemented with 1x MEM AA. J) Head-to-head comparison of 4 v/v\%

660 glycerol BMGY, 4 v/v\% glycerol rich defined medium, the initial defined biomass accumulation

661 media (DM1_dev0), and the final biomass accumulation medium obtained after a full

662 optimization cycle (DM1), demonstrates that DM1 leads to superior biomass accumulation. 


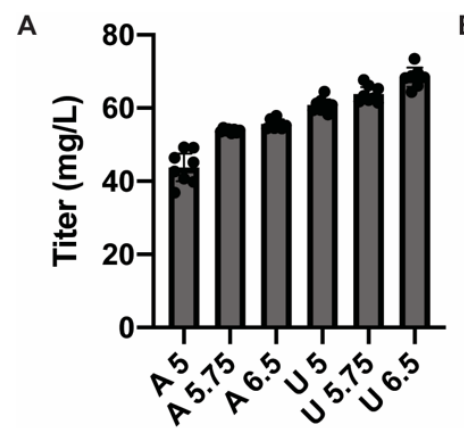

D

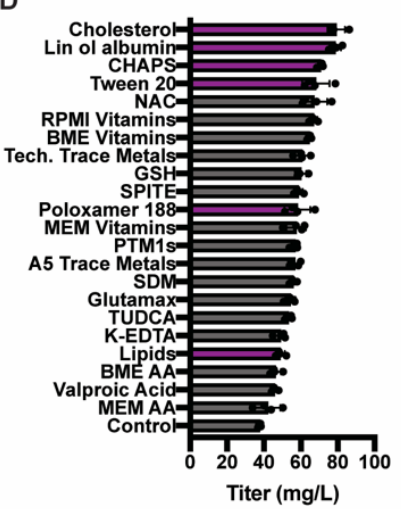

H

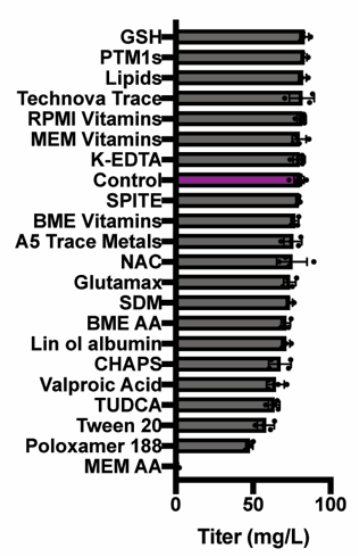

B

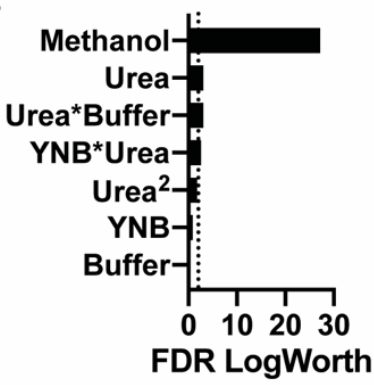

C

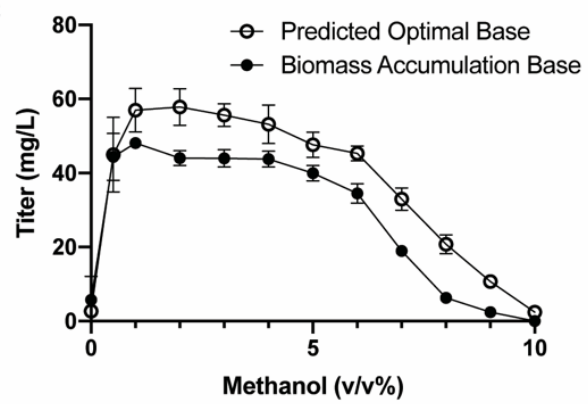

G

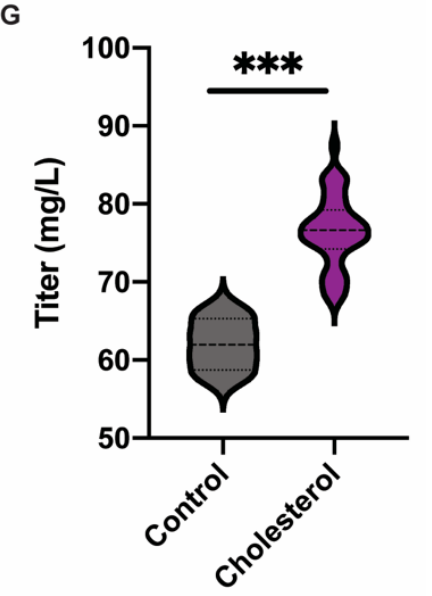

K

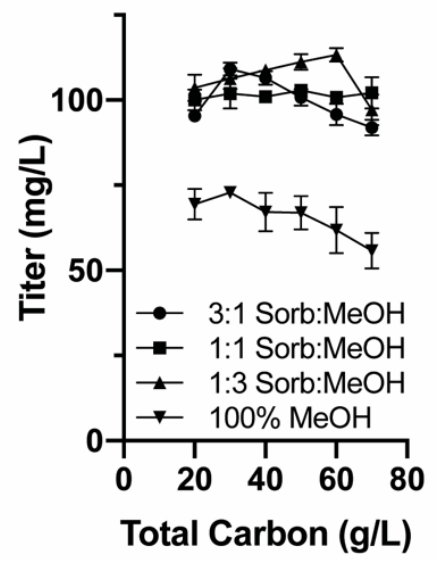

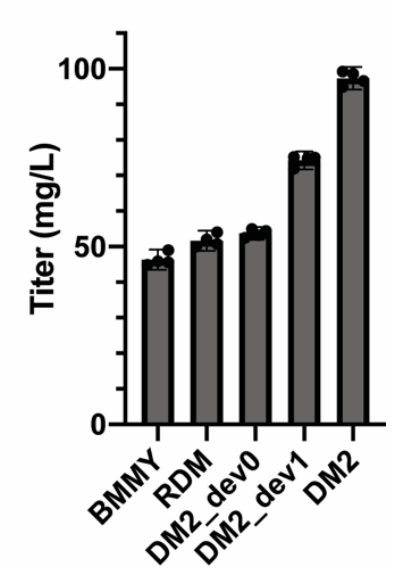

665
I

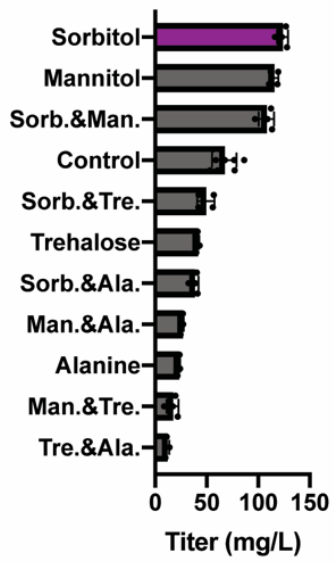

E

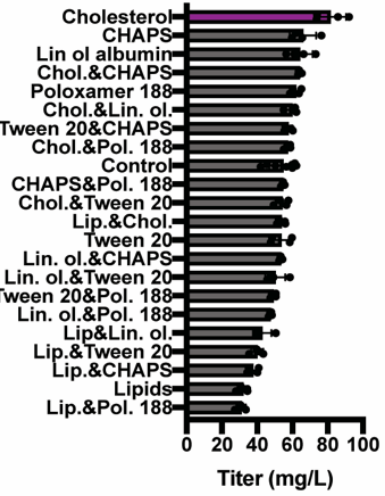

$\mathbf{J}$

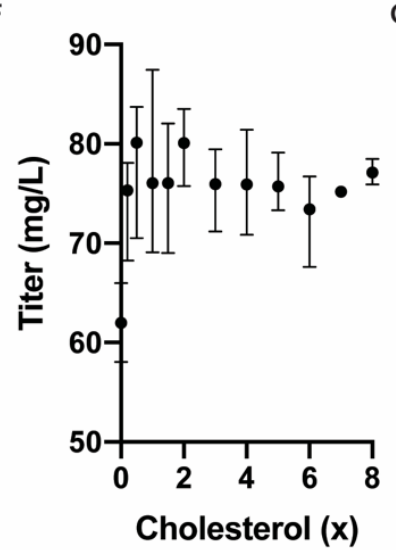

Figure 3. Modular development of a media for heterologous protein production in P. pastoris A) Initial full-factorial screen of nitrogen source choice and buffer $\mathrm{pH}$ demonstrates that urea is preferred over ammonium sulfates and high buffer $\mathrm{pH}$ is preferred over lower values. B) A fullfactorial concentration optimization identified methanol as the most concentration dependent variable. Other components in the base media were predicted to affect productivity with much lower levels of significance. C) Evaluation of the effect of methanol concentration on P[8] titer, using two different base media (urea, buffer, and YNB concentrations): the biomass 
673 accumulation base medium and the optimal base media composition predicted by our

674 concentration DOE. D) Ranking of supplements according to their effect on P[8] titer.

675 Supplements related to membrane fluidity or lipid metabolism ranked highly. E) Evaluation of

676 combinations of lipid and surfactant supplements confirmed that cholesterol supplementation

677 leads to the greatest improvement in P[8] titer. F) Concentration optimization of cholesterol

678 demonstrated low concentration dependence, with similar performance observed over a 40-fold

679 range (0.2-8x). G) Comparing cholesterol-free and cholesterol-supplemented cultures fed at

680 various concentrations demonstrates that cholesterol supplementation results in a significant

$681 \sim 25 \%$ improvement in $\mathrm{P}[8]$ titers $(\mathrm{p}<0.001)$. H) No significantly beneficial supplements were

682 observed when repeating the supplementation screen. I) Screening supplementation of $20 \mathrm{~g} / \mathrm{L}$

683 of co-fed substrates individually or in 1:1 combinations by mass identified sorbitol

684 supplementation as highly beneficial to P[8] titer. J) Examination of the effect of co-feed ratio

685 and total carbon concentration on titer in DM2_dev1 supplemented media. K) Comparison of

$686 \mathrm{P}[8]$ titer obtained with DM2 to previous iterations and other common $P$. pastoris media

687 demonstrates a $\sim 2 \mathrm{x}$ improvement in $\mathrm{P}[8]$ titer, relative to $1 \mathrm{v} / \mathrm{v} \%$ methanol RDM and $1 \mathrm{v} / \mathrm{v} \%$

688 methanol BMMY. 
A

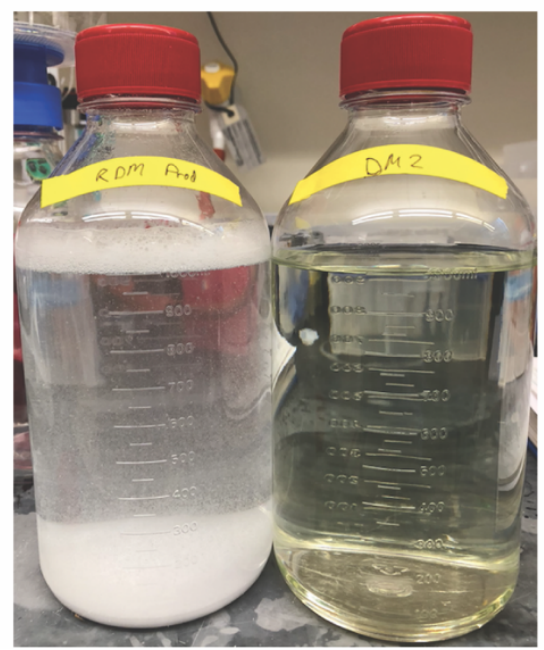

B

DM2 Volumetric Composition

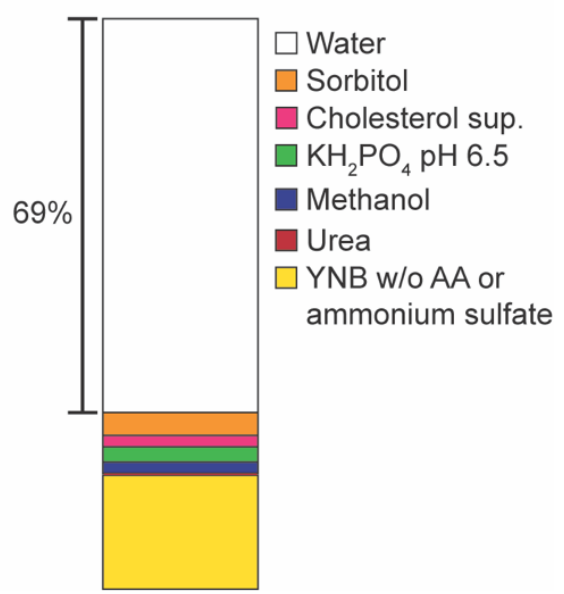

691 Figure 4. Comparison of DM2 to rich define medium.

692 A) Comparison of precipitate formation during construction of RDM (left) and DM2 (right) media.

693 Adjusting the $\mathrm{pH}$ of $\mathrm{RDM}$ to 6.5 results in significant formation of white precipitate. No

694 precipitate formation is observed in DM2. B) Relative volumes of stock solutions and pure water 695 needed to construct DM2. Pure water addition accounts for $69 \%$ of DM2 volume, demonstrating

696 that there is substantial room for further supplement exploration and development. When

697 separated into simple stock solutions, DM2 can be $3 x$ concentrated. 